\title{
The X-ray and radio states of Cyg X-3 - classification and long-term correlations
}

\section{A. Szostek}

Max Planck Institut für Kernphysik, Saupfercheckweg 1, Heidelberg 69117, Germany Astronomical Observatory, Jagiellonian University, Orla 171, 30-244 Kraków, Poland

E-mail: asz@oa.uj.edu.pl

\section{A. A. Zdziarski}

Centrum Astronomiczne im. M. Kopernika, Bartycka 18, 00-716 Warszawa, Poland

E-mail: aaz@camk.edu.pl

\section{L. McCollough}

Smithsonian Astrophysical Observatory, 60 Garden Street, MS 67, Cambridge, MA 02138-1516, US A

E-mail: mmccollough@head.cfa.harvard.edu

I will present detailed classification of the X-ray states of Cyg X-3 based on the spectral shape and a new classification of the radio states based on the long-term correlated behaviour of the radio and soft X-ray light curves. Except for the effect of strong absorption and the energy of the high-energy break in the hard state, the X-ray spectral states of Cyg X-3 closely correspond to the canonical X-ray states of black-hole binaries. Also, the radio X-ray correlation closely corresponds to that found in black-hole binaries, but it significantly differs from that in neutronstar binaries. Overall, our results strongly support the presence of a black hole in Cyg X-3.

VII Microquasar Workshop: Microquasars and Beyond September 1-5 2008

Foca, Izmir, Turkey

\footnotetext{
* Speaker.
} 


\section{Introduction}

Cyg X-3 is the brightest radio source among X-ray binaries [1]. It is a high-mass system with a Wolf-Rayet companion, but with an unusually short orbital period, $P=4.8 \mathrm{~h}$. It is located at a distance of $d \simeq 9 \mathrm{kpc}$ in the Galactic plane [2]. In spite of its discovery in 1966, the system remains poorly understood. In particular, due to the lack of a reliable mass function, it remains uncertain whether its compact object is a black hole or a neutron star.

As an X-ray source, Cyg X-3 is persistent and observed in two main spectral states, hard and soft. Recently, the hard state has been studied in detail by [3], and both the hard and soft states, by [4]. However, there has yet been no comprehensive studies of the range of the states including the transitionary ones. Interestingly, the spectra of Cyg X-3 in spite of being generally similar to the canonical states of black hole binaries show some significant differences [5]. Their physical interpretation also remains rather uncertain $[3,4]$. A major issue is the strong and complex intrinsic absorption, most likely caused by the wind from the companion star, which greatly complicates the determination of the intrinsic spectral shapes and luminosities. However, even detailed treatment of the wind absorption did not allow for an unambiguous determination of details of the underlying radiative processes in the two main spectral states [4]. Thus, it is highly desirable to further study the X-ray states of this system.

In the radio, Cyg X-3 is a persistent source in the sense that is always detected, though its flux varies by four orders of magnitude, as well as the relative contributions of the core and jets vary strongly (e.g., [6]). On the basis of 1988-1992 radio observations by the Green Bank Interferometer (GBI), [7-9] identified four of its radio states. They include the so-called quiescent radio emission, with a 50-200 mJy flux variable on a timescale of months, episodes of frequent minor flaring, $\leq 0.3 \mathrm{Jy}$, very weak, or quenched, emission, $\leq 30 \mathrm{mJy}$, and major flares, $\sim 1-20 \mathrm{Jy}$, which follow the quenched state. (Note that the term 'quiescent' used here is not related to the so-called quiescent state of low-mass X-ray binaries, which corresponds to much weaker states than the above one of Cyg X-3.) Interestingly all major radio flares were preceded by very low radio flux levels. However, apart from that sequence, those authors did not study either the temporal patterns of the radio states or their connection to the X-ray states.

The existence of a relationship between the two energy bands was first pointed out by [10]. They showed that strong radio flares occur only when the source is in its soft state. Then, [1] found an anticorrelation in the quiescent state (corresponding to the hard state in X-rays) between the radio emission and the hard X-ray, 20-100 keV. The two bands were positively correlated during major radio flares and in the quenched state. No correlation was observed during minor flares. $[3,11]$ have presented the correlation between the $1.5-12 \mathrm{keV}$ X-ray flux and the $15 \mathrm{GHz}$ measurements by the Ryle telescope. They found a clear positive correlation in the hard X-ray state, and its break down above certain X-ray flux. The sign of the correlation of the 1.5-12 keV flux is opposite to that of the $20-100 \mathrm{keV}$ flux, which is consistent with the anticorrelation between those two X-ray bands $[3,12]$. However, details of the radio/X-ray behaviour in the soft state were not clear from these works.

Here we present a detailed classification of the X-ray spectral states based on the X-ray data from the RXTE Proportional Counter Array (PCA) and the High Energy X-Ray Transient Experiment (HEXTE). We re-classify the radio states based the long-term temporal behaviour of the GBI 
(radio) and RXTE ASM (soft X-ray) light curves, and relate these states to the X-ray spectral states. Also, we analyze correlations of hard X-rays with the other bands using data from the BATSE. Finaly, we summarize and discuss our results, as well as compare them to the corresponding results for black-hole and neutron-star X-ray binaries.

\section{The data}

In our analysis, we use the 3-5 keV soft X-ray band of the RXTE ASM dwell data and the 20-100 keV hard X-ray data from the BATSE which have been obtained using an Earth occultation analysis technique [13]; see [1] for analysis of an earlier part of the BATSE data. Each point represents a 3-day average of the energy flux. The radio data have been collected during the monitoring program of the GBI [7-9].

We then rebin the ASM and GBI light curves in such way that the significance of a single bin is $\geq 3 \sigma$. The rebinned data are also plotted in the radio/soft X-ray correlation diagrams requiring each pair of points to be taken on the same day. Given the relatively low statistical quality of the BATSE data, we rebin its light curve to $\geq 8 \sigma$ significance (but requiring the distance between the midpoints of the data in a given bin to be $\leq 10$ days). In showing correlations of the hard X-ray data with the radio and soft X-rays, we slightly modify this criterion in order to achieve the best clarity of the graphical representations. Also, since the measured fluxes from the BATSE are often null, we use a linear scale for it (while we use logarithmic scales for all other data).

We also use selected RXTE PCA and HEXTE pointed observations. We have selected 42 pointed observations from 1996-2000 for which the PCA data from the Proportional Counter Units 0-2 were available. We have extracted the PCA spectra in the 3-25 keV range using the top layer of the detectors. We then obtained the corresponding HEXTE spectra, using both HEXTE clusters, in the $15-110 \mathrm{keV}$ energy band. In our spectral fits, we allow a free relative normalization of the HEXTE spectra with respect to that from the PCA.

\section{The X-ray spectral states}

We fit each of the $42 \mathrm{PCA} / \mathrm{HEXTE}$ spectra with the model as applied to INTEGRAL/RXTE spectra from Cyg X-3 by [14]. Similar models were also used by [3, 4]. The model includes Comptonization by hybrid (i.e., both thermal and nonthermal) electrons [15, 16], Compton reflection from an ionized medium [17], absorption by fully and partially covering neutral media, and a Gaussian Fe K fluorescent line. As discussed in [14], this model treats the low-energy part of the spectrum only phenomenologically. Still, it provides good fits to the PCA/HEXTE spectra and a physical description of the hard X-rays.

The obtained (absorbed) model spectra are shown in Fig. 1(a). We have divided them into five groups based on their spectral shape and ordered by the decreasing flux at $20 \mathrm{keV}$. The increasing group number also roughly corresponds to the decreasing spectral hardness in the $10-20 \mathrm{keV}$ range. The groups 1-2, 3-4, and 5 may be classified as belonging to the hard, intermediate/soft and ultrasoft state, respectively. Some of the flux variability within each group is caused by the orbital modulation. 

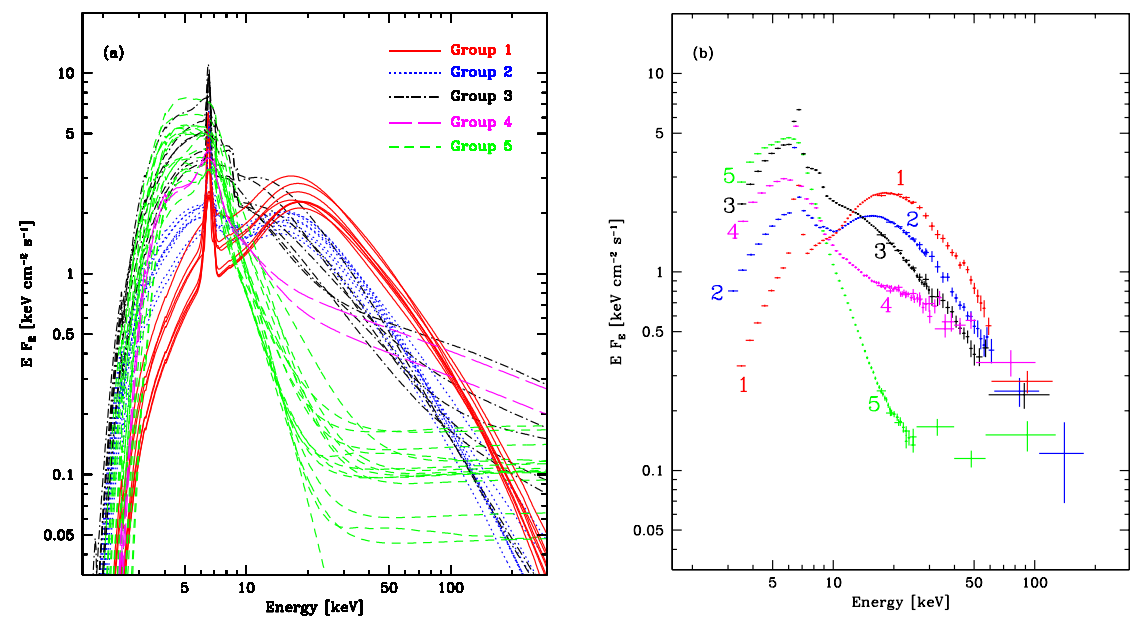

Figure 1: (a) Comptonization model spectra of Cyg X-3 from the 42 pointed $R X T E$ observations. Different line styles and colours correspond to our classification of the spectra. (b) Deconvolved spectra for the average of each of the 5 groups shown in the panel (a), fitted with the same model as the individual spectra. The HEXTE data are renormalized to the level of the PCA.

We have then created the average PCA/HEXTE spectra within each group. We have fitted them with the same model as above, obtaining $0.3<\chi_{v}^{2}<1.9$ (with a 1 per cent systematic error added in quadrature to the statistical error). The resulting spectra are shown in Fig. 1(b). The resulting isotropic luminosities for groups $1,2,3,4$ and 5 are $7.5 \times 10^{37}, 7.5 \times 10^{37}, 1.1 \times 10^{38}$, $7.9 \times 10^{37}$ and $7.9 \times 10^{37} \mathrm{erg} \mathrm{s}^{-1}$ respectively (for an assumed distance of $9 \mathrm{kpc}$ ).

Both [3] and [4] found relatively high fractions of nonthermal electrons in the hard state, which is in contrast to the hard state of black-hole binaries being usually dominated by thermal electrons, e.g., $[5,18]$. The intermediate and soft-state spectra are dominated by the disc component, but then followed by a significant hard tail clearly requiring the presence of both hot thermal electrons and nonthermal ones, similarly to the soft states of black-hole binaries, e.g., [5, 18].

\section{Radio emission and its correlation with X-rays}

Here, we revisit the classification of the radio states of [7-9]. We consider here the 8.3 $\mathrm{GHz}$ radio light curve together with those of $3-5 \mathrm{keV}$ and $20-100 \mathrm{keV} \mathrm{X}$-rays, see Fig. 2. From joint analysis of the $8.3 \mathrm{GHz}$ and 3-5 keV light curves, we have found the following six distinct variability patterns, with four of them corresponding to the previous classification. They are related to a transition level in both fluxes, shown by the horizontal dotted line in Fig. 2.

1. The quiescent state: both the radio and soft X-ray fluxes vary in a correlated manner below the transition level.

2. The minor-flaring state: the soft X-ray flux oscillates around its transition level while the radio flux varies up to its transition level.

3. The suppressed state: the radio flux is below the transition level and the soft X-ray flux is above it, and the state is not directly followed by a radio flare. 


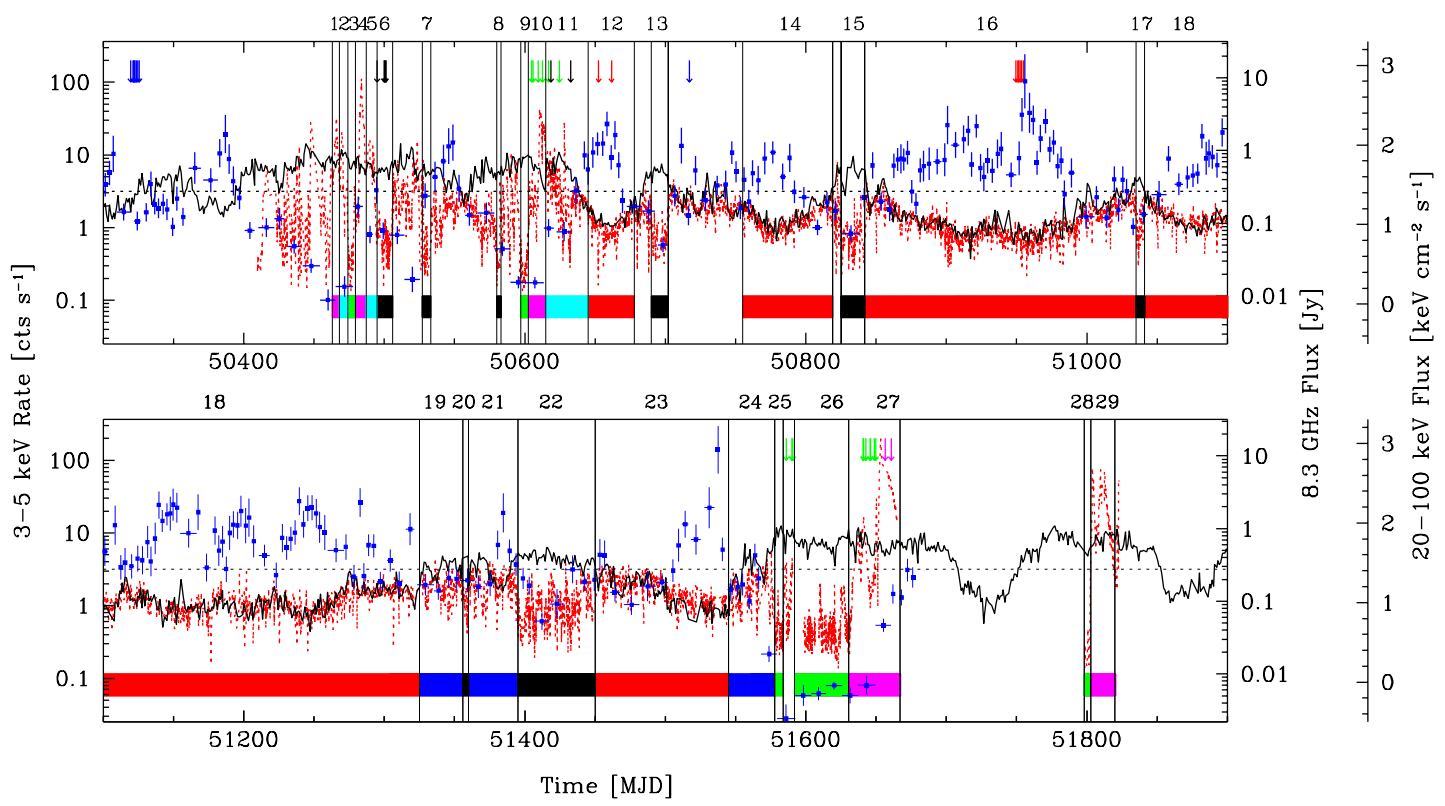

Figure 2: The RXTE ASM 3-5 keV, BATSE 20-100 keV, and GBI $8.3 \mathrm{GHz}$ light curves, shown as the black solid curves, blue crosses, and red dotted curves, respectively. The horizontal dotted line represents the transition level at which the radio/soft X-ray correlation changes its character. The vertical lines divide the light curves into intervals of different activity types. The patterns of similar behaviour are identified by the colours of the boxes and the number on top of the intervals. Intervals 12, 14, 16, 18 and 23 correspond to quiescence, 19, 21 and 24 to minor flaring, 6, 7, 8, 13, 15, 17, 20 and 22 to suppressed, 3, 9, 25, 26 and 28 to quenched, 1, 4, 10, 27 and 29 to major flaring and 2, 5 and 11 to post-flare. Note that some intervals have remained unclassified. The arrows show the times of the pointed PCA/HEXTE observations, and their colours identify the spectral states shown in Figs. 1.

4. The quenched state: the radio flux is much below the transition level and the soft X-ray flux is above it, and the state is followed by a major radio flare.

5. The major-flaring state: the soft X-ray flux is above the transition level, and the radio flux moves up and down by a large factor in a flare.

6. The post-flare state: the return after a major flare to either the minor-flaring state or the suppressed one.

The transition level corresponds to the $3-5 \mathrm{keV}$ count rate of $\simeq 3 \mathrm{~s}^{-1}$, and the $8.3 \mathrm{GHz}$ flux of $\simeq 0.3 \mathrm{Jy}$. It defines a boundary above which the positive correlation between the two fluxes breaks down. The radio transition level represents an approximate upper limit to the radio flux in most states, and it is crossed mostly during strong flares. The vertical solid lines in Fig. 2 divide intervals corresponding to different states.

Fig. 3 presents the correlation between each two fluxes for the intervals with the identified variability pattern, marked by different colours. In Fig. 3(a) the six different states form a clear sequence of correlated radio/X-ray behaviour, with the sequence of the quiescent, minor-flaring, suppressed, quenched, major-flaring, and post-flare states. Combining the information obtained from pointed observations shown in Fig. 1 and lightcurves plotted in Fig. 2 we found out that the 

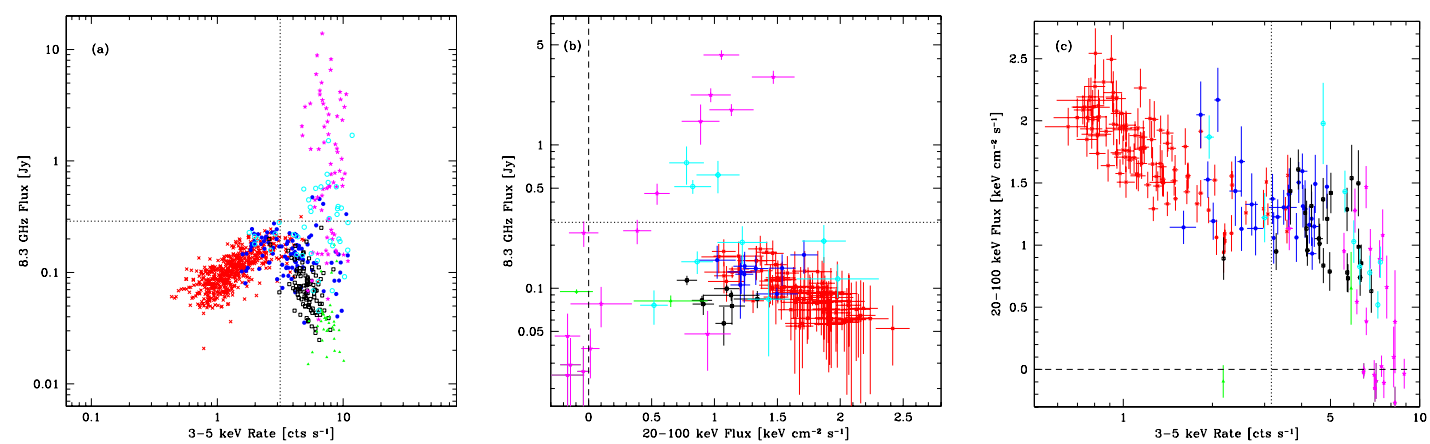

Figure 3: (a) The $8.3 \mathrm{GHz}$ radio flux as a function of the ASM 3-5 keV count rate. The red crosses, blue filled circles, black open squares, green filled triangles, magenta stars and cyan open circles correspond to the quiescent, minor-flaring, suppressed, quenched, major-flaring, and post-flare states, respectively, see Fig. 2. The dotted lines represent the X-ray and radio transition levels shown in Fig. 2. (b) The GBI flux as a function of the BATSE flux. The dashed line gives the zero BATSE flux and the dotted line corresponds to the soft X-ray transition level defined in Fig. 2. (c) The BATSE flux as a function of the ASM flux. The colours on panels (a) and (b) correspond to the radio states in panel (a).

hardest X-ray state, type 1, corresponds to the quiescent radio state. There were no GBI measurements corresponding to most of the X-ray spectral type 2 observations. Thus, we have used instead the $15 \mathrm{GHz}$ Ryle data (presented in [3]) and concluded that this X-ray state corresponds to the minor-flaring state. Most of the pointed observations corresponding to the X-ray type 3 correspond to the suppressed state. Then the X-ray types 4 and 5 take place during the major-flaring state. There were no pointed observations corresponding to the quenched state.

The sequence of radio and X-ray states we have found is as follows. The point where radio and X-ray transition levels, shown in Fig. 2, cross, govern much of the observed behavior. To the left of this point, Cyg X-3 is in the radio quiescent state and in the hard X-ray state (type 1). In the quiescent/hard state, the source moves up and down along this branch, and the radio emission is correlated with soft X-rays. The minor-flaring state (X-ray type 2) corresponds to a transition between the radio quiescent state and the suppressed state, as well as between the hard and soft Xray states. These transitions may occur in both directions, without any apparent hysteresis effects (unlike black-hole transients, but similarly to Cyg X-1, [5]). The radio/X-ray correlation changes its sign in this state from positive to negative. Consequently, a further increase of the X-ray flux corresponds to a decrease of the radio flux, first to the suppressed state and then to the quenched one. As mentioned above, the suppressed state is not necessarily followed by a radio flare. From this state, the source has still been observed to return to the quiescent/hard state through the minorflaring one. If, however, the X-ray and radio fluxes keep increasing and decreasing, respectively, Cyg X-3 enters the quenched state. At this point, the $3-5 \mathrm{keV}$ and $8.3 \mathrm{GHz}$ fluxes achieve their overall maximum and minimum, respectively. In both the suppressed and quenched states, the radio emission is anticorrelated with the $3-5 \mathrm{keV} \mathrm{X}$-rays.

Interestingly, all major radio flares have been observed only after a radio quenched period, and in all cases except one (the interval 25 in Fig. 2, in which case after some initial increase of the radio flux the source returned to the quenched state, interval 26), the quenched state is followed by a 

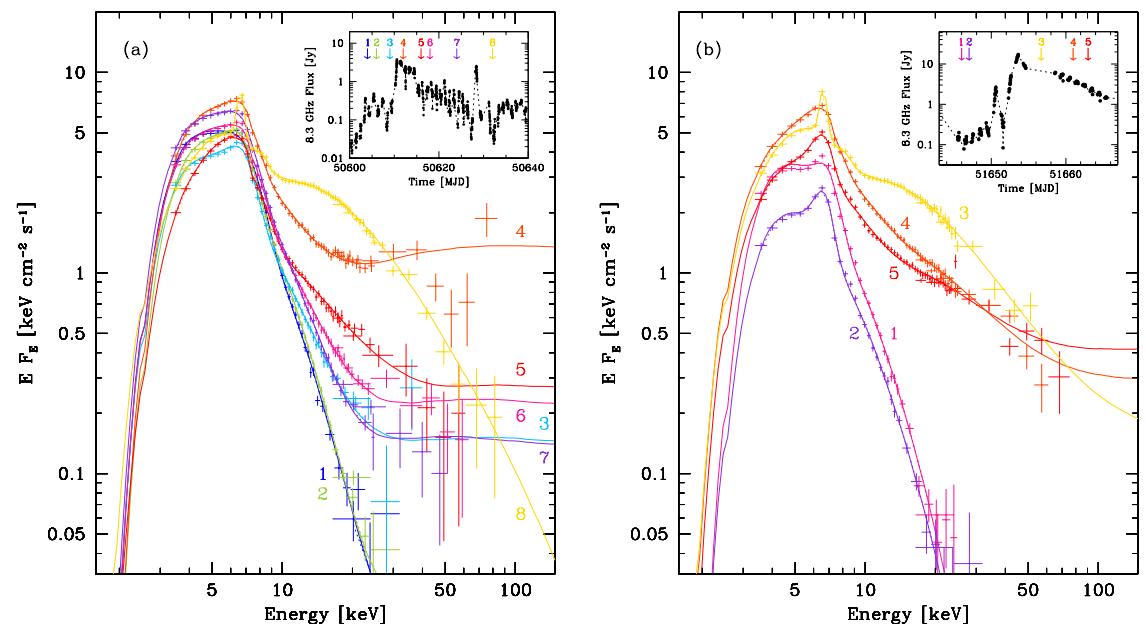

Figure 4: The PCA/HEXTE spectra taken during two major radio flares. The numbers correspond to the increasing time, and are identified in the insets, giving the radio light curves.

major flare. While on the major-flaring branch, the radio flux changes by more than three orders of magnitude while at the same time the 3-5 keV flux remains almost constant, and the X-ray spectra belong to the types 4,5 . The decline of the radio flux after a flare is through the minor-flaring and suppressed states, as shown in Fig. 3(a), but not through the quenched state. Thus, we see a hysteresis in this type of transitions, with the source following a loop in the flux-flux diagram. The post-flare stage is not strictly a new state but it is defined by its temporal appearance after a major flare.

We now compare the radio/soft X-ray correlation with that for hard X-rays. Fig. 2 shows the 20-100 keV BATSE light curve. Fig. 3(b) shows the $8.3 \mathrm{GHz}$ flux as a function of the 20-100 keV flux. We see an anticorrelation in the quiescent/hard state, which changes into a positive correlation on the major-flaring branch (including a few points from the post-flare state). This behaviour is the same as that found by [1] for an earlier data set. The two correlations form two clear branches. The overall evolutionary track is from the quiescent state to the minor flaring, where the negative correlation already changes sign, and both the radio and the hard X-ray fluxes drop to the quenched state. Then, they increase again along the positive correlation, see Fig. 3(b). The return from a major flare avoids the quenched state and it goes directly to the minor flaring (or, sometimes, suppressed) state, as shown by the cyan points. Fig. 3(c) shows the corresponding ASM/BATSE diagram. There is a strong anticorrelation in the hard/quiescent state, after which the $20-100 \mathrm{keV}$ flux becomes independent of the $3-5 \mathrm{keV}$ one until the major-flaring state, in which the correlation becomes again negative and the $20-100 \mathrm{keV}$ flux drops to zero.

Fig. 4 presents two series of the PCA/HEXTE spectra taken during the radio flares that started at MJD $\sim 50610$ and $\sim 51650$. The spectra were fitted with the same model as in Section 3. We see that the hard tail flux varies much more than that of the soft X-rays. The flares start with ultrasoft spectra with no high-energy tail and then the tail strength increases, going through a sequence of the X-ray spectral types $5,4,3$. Thus, the strength of the high-energy tail is positively correlated with the radio flux, as we have already seen in Fig. 3(b). 


\section{Discussion and comparison to other X-ray binaries}

We find that overall the radio/soft X-ray correlation in Cyg X-3 is rather similar to those of black-hole binaries. In the hard state, the positive correlation in Cyg X-3 is very similar to that of black-hole binaries in the hard state [11, 19-21]. When Cyg X-3 enters softer states from the hard state, the radio flux starts to decline and the correlation changes its sign. This is again similar to other black hole binaries. In particular, the radio flux strongly declines at the transition from the hard state to a soft one in Cyg X-1 (e.g., [11]), GX 339-4 [19], XTE J1650-500 [20], and XTE $\mathrm{J} 1859+226$ [21]. After the decline, the radio flux from black-hole transients strongly increases, sometimes up to the overall maximum for a source, see [21]. This behaviour is also seen in the black-hole sample shown in fig. 7 of [22]. A very bright radio flare of GX 339-4 took place during a transition from the hard state to a soft (very-high) one [23], which typifies the above pattern. Although Cyg X-1, a persistent source, does not generally follow the whole of the above pattern (which appears to be due to the limited range of luminosities it covers), it also shows episodes of a radio flux in its soft state as strong as in the hard state, see, e.g., its radio light curve in [24]. The two brightest observed radio flares of Cyg X-1 happened in intermediate/soft spectral states [25, 26]. As mentioned above, GRS $1915+105$ is never in the hard state, and its radio/X-ray flux evolution resembles the quenched/major-flare branch of Cyg X-3, see [11, 27]. All of those similarities provide a very strong argument for the black-hole nature of the compact object in Cyg X-3.

Given the hard-state anticorrelation between the soft and hard X-rays, the positive correlation of the radio flux with the soft X-rays becomes a negative one with the hard X-rays, see Fig. 3(b). The fact that the correlation of the radio emission is positive with the soft X-rays points out to the importance of the blackbody-emitting disc (or its inner edge) to the jet formation in the hard state.

On the other hand, while the soft X-ray flux is almost constant during major flares, the hard $\mathrm{X}$-ray flux is strongly (and positively) correlated with the radio flux during major flares. The X-ray spectra during the major flares are soft/high, very high, or ultrasoft (Fig. 4). The dominant spectral components during major flares in the $3-5 \mathrm{keV}$ and $20-100 \mathrm{keV}$ bands are the weakly varying (disc) blackbody and a strongly varying high-energy tail, respectively, see Fig. 4. This behaviour is likely explained by the presence of a stable accretion disc and variable active regions above the disc, and it is again typical to black-hole binaries, see the analogous result for the soft state of Cyg $\mathrm{X}-1$ in [28]. In the case of Cyg X-3, we have discovered that the radio flux during major flares responds exponentially to the amplitude of the high-energy tail, see Fig. 3(b). This shows that the radio-emitting region communicates, on time scales of the order of a day, with the X-ray source, and it is not detached from it.

An important issue is the origin of the high-energy, hard X-ray, tail in the soft states. [16, $28-$ 31] modelled this tail in the black-hole binaries Cyg X-1, GRS 1915+105, and XTE J1550-564 by Comptonization in a hybrid plasma, in which the electron distribution contains a significant nonthermal high-energy tail. This Comptonization presumably takes place in some coronal regions above the accretion disc. The correlation of the tail amplitude with the radio flux may be then due to the formation of radio-flare emitting blobs being related to the non-thermal coronal regions. Still, the observed radio flux has to be due to synchrotron emission in jet regions relatively far away from the compact object, which is required by the lack of any orbital modulation in Cyg X-3 by 
free-free absorption in the stellar wind [32].

On the other hand, a rather common model in the astrophysical literature attributes hard X-ray tails of black-hole binaries in soft states directly to emission of non-thermal electrons in the jet, usually via the synchrotron process. There are two major problems with this model (see [33]). One is that the spectra of the tails are generally soft, with the photon indices of $\Gamma \geq 2$, typically $\sim 3$, which is also the case in most of the soft states of Cyg X-3 (Fig. 1). Such a soft spectrum extrapolated to the turnover frequency, typically in the infrared, would yield a huge luminosity, orders of magnitude higher than those observed. Second, the high-energy tails are always smoothly connected to the disc component, which is naturally explained by the tails being due to Comptonization of the disc photons. If they were due to a process unrelated to the disc, their amplitude in the hard X-ray region could be any, without any reason for this smooth connection. The first problem can be circumvented by requiring that the synchrotron spectrum is hard at low energies and it breaks to a soft one just below the region where the tails are observed. This, however, requires yet another fine-tuning, and given the large observed sample of high-energy tails, can be ruled out on statistical grounds.

Finally, we point out that the radio source in Cyg X-3 consists of the core and the jet components, which can be resolved (e.g., [34, 35]. In fact, the e-VLBI results of [6] show that the core may become undetectable during a radio flare. On the other hand, our analysis in the radio band has been done using data from the GBI, which beam includes both the core and jet components, which did not allow us to include the spatial dimension in the correlation analysis. Also, our analysis did not take into account time lags of the radio emission with respect to the $\mathrm{X}$-rays. These issues, as well as development of theoretical models, should be dealt with in future studies of radio/X-ray correlations.

More on the presented correlations can be found in [36].

\section{Acknowledgments}

This research has been supported in part by the Polish MNiSW grant NN203065933 (20072010) and the Polish Astroparticle Network 621/E-78/SN-0068/2007. The GBI was operated by the National Radio Astronomy Observatory for the U.S. Naval Observatory and the Naval Research Laboratory during the the observations used here.

\section{References}

[1] M. L. McCollough, et al. ApJ 517, 951 (1999)

[2] J. M. Dickey, ApJ 273, L71 (1983)

[3] L. Hjalmarsdotter, A. A. Zdziarski, S. Larsson, V. Beckmann, M. L. McCollough, D. C. Hannikainen, O. Vilhu, MNRAS 384, 278 (2008)

[4] A. Szostek, A. A. Zdziarski, MNRAS 386, 593 (2008)

[5] A. A. Zdziarski, M. Gierliński, Progr. Theor. Phys. Suppl. 155, 99 (2004)

[6] V. Tudose, et al., MNRAS 375, L11 (2007)

[7] E. B. Waltman, R. L. Fiedler, K. L. Johnston, F. D. Ghigo, AJ 108, 179 (1994) 
[8] E. B. Waltman, F. D. Ghigo, K. J. Johnston, R. S. Foster, R. L. Fiedler, J. H. Spencer, AJ 110, 290 (1995)

[9] E. B. Waltman, R. S. Foster, G. G. Pooley, R. P. Fender, F. D. Ghigo, AJ 112, 2690 (1996)

[10] H. Watanabe, S. Kitamoto, S. Miyamoto, R. L. Fielder, E. B. Waltman, K. J. Johnston, F. D. Ghigo, ApJ 433, 350 (1994)

[11] E. Gallo, R. P. Fender, G. G. Pooley, MNRAS 344, 60 (2003)

[12] M. Choudhury, A. R. Rao, S. V. Vadawale, C. H. Ishwara-Chandra, A. K. Jain, AEFA 383, L35 (2002)

[13] B. A. Harmon, et al., ApJS, 138, 149 (2002)

[14] O. Vilhu, et al., AEFA 411, L405 (2003)

[15] P. S. Coppi, in J. Poutanen, R. Svensson, eds., ASP Conf. Ser. Vol. 161, High Energy Processes in Accreting Black Holes, p. 375 (1999)

[16] M. Gierliński, A. A. Zdziarski, J. Poutanen, P. S. Coppi , K. Ebisawa, W. N. Johnson, MNRAS 309, 496 (1999)

[17] P. Magdziarz, A. A. Zdziarski, MNRAS 273, 837 (1995)

[18] M. L. McConnell, et al., ApJ 572, 984 (2002)

[19] S. Corbel, R. P. Fender, A. K. Tzioumis, M. Nowak, V. McIntyre, P. Durouchoux, R. Sood, AEA 359, 251 (2000)

[20] S. Corbel, R. P. Fender, J. A. Tomsick, A. K. Tzioumis, S. Tingay, ApJ 617, 1272 (2004)

[21] R. P. Fender, T. M. Belloni, E. Gallo, MNRAS 355, 1105 (2004)

[22] A. Merloni, S. Heinz, T. Di Matteo, MNRAS 345, 1057 (2003)

[23] E. Gallo, S. Corbel, R. P. Fender, T. J. Maccarone, A. K. Tzioumis, MNRAS 347, L52 (2004)

[24] P. Lachowicz, A. A. Zdziarski, A. Schwarzenberg-Czerny, G. G. Pooley, S. Kitamoto, MNRAS 368, 1025 (2006)

[25] R. P. Fender, A. M. Stirling, R. E. Spencer, I Brown, G. G. Pooley, T. W. B. Muxlow, J. C. A. Miller-Jones, MNRAS 369, 603 (2006)

[26] J. Wilms, K. Pottschmidt, G. G, Pooley, S. Markoff, M. A. Nowak, I Kreykenbohm, R. E. Rothschild, ApJ 663, L97 (2007)

[27] M. Klein-Wolt, R. P. Fender, G. G. Pooley, T. Belloni, S. Migliari, E. H. Morgan, M. van der Klis, MNRAS 331, 745 (2002)

[28] A. A. Zdziarski, J. Poutanen, W. S. Paciesas, L. Wen, ApJ 578, 357 (2002)

[29] A. A. Zdziarski, J. E. Grove, J. Poutanen, A. R. Rao, S. V. Vadawale, ApJ 554, L45 (2001)

[30] A. A. Zdziarski, M. Gierliński, A. R. Rao, S. V. Vadawale, J. Mikołajewska, MNRAS 360, 825 (2005)

[31] M. Gierliński, C. Done, MNRAS 342, 1083 (2003)

[32] A. Szostek, A. A. Zdziarski, MNRAS 375, 793 (2007)

[33] A. A. Zdziarski, P. Lubiński, M . Gilfanov, M. Revnivtsev, MNRAS 342, 355 (2003)

[34] J. Martí, J. M. Paredes, M. Peracaula M.,ApJ 545, 939 (2000)

[35] A. J. Mioduszewski, M. P. Rupen, R. M. Hjellming, G. G. Pooley, E. B. Waltman, ApJ 553, 766 (2001)

[36] A. Szostek, A. A. Zdziarski, M. L. McCollough, MNRAS, 388 1001, (2008) 